See discussions, stats, and author profiles for this publication at: https://www.researchgate.net/publication/250144398

\title{
Identification and Separation of Cannabis sativa, Embleia ribes, Myristica fragrans and Piper longum from Organic Extract on Silica Gel Surface with Anionic Micellar Solvent System...
}

Article in The Open Nutraceuticals Journal · May 2010

DOI: $10.2174 / 1876396001003030112$

CITATIONS

4

4 authors, including:

Abdalkader salih Mohammad

Universidad de Monterrey

25 PUBLICATIONS 154 CITATIONS

SEE PROFILE

Ge Ram Kushal Singh

1. Deen Dayal Upadhyay Gorakhpur University

511 PUBLICATIONS 9,825 CITATIONS

SEE PROFILE

Some of the authors of this publication are also working on these related projects:

Contribution of Dr R K Singh in Sugarcane View project

Project Nutrition in Health and Disease View project
READS

54

Showkat Bhawani

University Malaysia Sarawak

48 PUBLICATIONS 263 CITATIONS

SEE PROFILE 


\title{
Identification and Separation of Cannabis sativa, Embleia ribes, Myristica fragrans and Piper longum from Organic Extract on Silica Gel Surface with Anionic Micellar Solvent System: Application in Ayurvedic Medicine
}

\author{
A. Mohammad ${ }^{1, *}$, S. Sharma ${ }^{2}$, S.A. Bhawani ${ }^{3}$ and Ram B. Singh ${ }^{4}$ \\ ${ }^{1,2,3}$ Analytical Research Laboratory, Department of Applied Chemistry, Faculty of Engineering \& Technology, Aligarh \\ Muslim University, Aligarh-202002, India \\ ${ }^{4}$ Halberg Hospital and Research Institute, Civil Lines Moradabad-10, (U.P), India
}

\begin{abstract}
Cannabis sativa, Myristica fragrans, Piper longum and Embleia ribes are the active pharma ingredients of an ayurvedic herbal formulation Jatiphaldya, which is beneficial in many typical stomach related disorders.

The present study is aimed to develop a simple and reliable thin layer chromatographic (TLC) method using micellar solution of sodium dodecyl sulfate (SDS) as mobile phase for the identification of all the four herbal drugs with preliminary separation on silica gel 'G' TLC plate. The active components of drug were extracted in a mixture of ethanol and water (4:1), chromatographed on silica gel TLC plate using aqueous SDS (5\%) as mobile phase and the resolved spots for Cannabis sativa $\left(\mathrm{R}_{\mathrm{F}}-0.95\right)$, Myristica fragrans $\left(\mathrm{R}_{\mathrm{F}}-0.64\right)$, Piper longum $\left(\mathrm{R}_{\mathrm{F}}-0.41\right)$ and Embleia ribes $\left(\mathrm{R}_{\mathrm{F}}-0.26\right)$ were identified using vanillin-sulfuric acid (2\% solution of vanillin in 5\% methanolic sulfuric acid). In order to realize most favorable mobile phase system in combination with silica gel ' $G$ ' as stationary phase, the effect of nature of surfactants (anionic, cationic or nonionic) and the level of concentration of each surfactant [sodium dodecyl sulfate (SDS), N-cetyl-N, $\mathrm{N}, \mathrm{N}$-trimethylammonium bromide (CTAB) or t- octyl phenoxydacaethoxy ethanol (TX-100)] on the mobility of all four active components was examined. In addition, the effect of organic (urea and alkanols) and inorganic $(\mathrm{NaCl})$ additives in 5\% aqueous SDS solution on mobility pattern of active herbal pharma ingredients was examined. The presence of ammonia and nitrate in the drug sample was found to hamper the resolution and identification of all active components. However, $\mathrm{NaCl}, \mathrm{KCl}$ and glucose do not offer serious interference.
\end{abstract}

Key Words: Thin layer chromatography, herbal, jatiphaladya, ayurvedic, surfactant.

\section{INTRODUCTION}

Jatiphaladya is a powdered herbal formulation of 6000 year old Indian ayurvedic system of medicine. Therapeutically Jatiphaladya is beneficial in many human intestinal disorders like tastelessness (aruci), Diarrhea (Atisara), Malabsorption syndrome (Graha), Dysentery (Pravahika), Cough (Kasa) and Rhinitis (Vatsale-ma-Pratisyaya). The main constituents of Jatiphaladya formulation are Cannabis sativa (Bhang), Myristica fragrans (Jatiphala), Piper longum (Pippali) and Embleia ribes (Vidanga) [1]. Cannabis sativa (Bhang) is a major constituent of the Jatiphaladya formulation $(20 \% \mathrm{wt} / \mathrm{vol})$. It is a commonly grown herb of high medicinal importance [2]. Myristica fragrans (Jatiphala) commonly known as nutmeg, is a popular herbal plant used in traditional Ayurvedic, Chinese and Thai medicine as aphrodisiac, antimicrobial, antipyretic, abortifacient and stomachic [3]. This plant is pharmacologically studied for various activities like chemo protective,

*Address correspondence to this author at the Analytical Research Laboratory, Department of Applied Chemistry, Faculty of Engineering \& Technology, Aligarh Muslim University, Aligarh-202002, India;

E-mail: alimohammad08@gmail.com antioxidant, aphrodisiac, antimicrobial, hepatoprotective, and anti-inflammatory [4]. Piper longum (Pippali) is a widely available species of the genus piper. Because of high therapeutic values, pipali has been used as antibacterial, anti-inflammatory and as a local anesthetic [5]. Embleia ribes (Vidanga) is a climbing herb found in Sri-lanka, India and Singapore. Therapeutically Embeleia inhibited pregnancy, possessed anti-estrogenic and weak progestational activity, shows analgesic, anti-inflammatory, antibacterial, antitumor and free radical scavenging activities $[6,7]$. As per our literature survey there is no analytical study available for Jatiphaladya formulation, But Previously Cannabis was analysed by various analytical methods including immunoassays (EMIT, Elisa fluorescence polarization, radioimmunoassay) [8], Gas Chromatography- Mass spectrometry [9], High performance liquid chromatographyMass spectrometry [10] and TLC [11]. However in case of Myristica fragrans (Jatiphal), Piper longum (Pippali) and Embleia ribes (Vidanga) a very few analytical studies are available using GC or HPLC [12-14]. Most of these techniques involves costly instruments and require highly purified standard reference samples [15]. Thin Layer Chromatography is a suitable method to screen simultaneously numerous samples extracted directly from 